\title{
Obturator Muscle
}

National Cancer Institute

\section{Source}

National Cancer Institute. Obturator Muscle. NCI Thesaurus. Code C33193.

One of two muscles located in the pelvis: the obturator externus muscle or the obturator internus muscle. 